\title{
A NOVEL APPROACH FOR HORIZONTAL AUGMENTATION OF POSTERIOR MAXILLA: TECHNICAL NOTE
}

\author{
Nazife Begüm KARAN*, Hüseyin Ozan AKINCI** \\ * DDS, PHD, Assistant Professor, Recep Tayyip Erdogan University, Faculty of Dentistry, Department of Oral And \\ Maxillofacial Surgery, Rize, Turkiye \\ ** DDS, PHD Resident, Gazi University, Faculty of Dentistry, Department of Oral and Maxillofacial Surgery, Ankara, \\ Turkiye
}

Over the years, there have been a series of innovative approaches to the alveolar bone augmentation techniques. These have led to the modifications of the existing methods and the establishment of more efficient ways to obtain sufficient bone mass that is necessary for the implant procedure. The aim of this technical note is to propose a novel augmentation technique, and investigate its efficacy, particularly during the healing process of the maxillary alveolar bone prior to the implant treatment.

\section{INTRODUCTION}

The use of dental implants is a highly favored treatment option with cumulative survival rates of over $90 \%$ in restoring partially or fully edentulous patients over the last decade. Although many technological advancements are present in implant dentistry, maintaining the sufficiency of the targeted bone area still remains to be the main necessity, and the challenge, for a successful implant placement [1].

Studies showed that, in the aftermath of tooth extraction the bone loses more than $40 \%$ of its volume in the first year. Besides, the vertical and horizontal resorptions significantly affect the amount of the residual bone mass that is necessary to place the implants [2]. Hence, the insufficiency of the alveolar bone area, generates the necessity to increase the bone volume in order to form adequate space to place implants. To this day, a number of surgical techniques and different graft materials have been developed to establish a more efficient methodology for the augmentation of the alveolar bone. Autogenous grafts currently provide the most widely used surgical treatment option that could be chosen to achieve the desired bone volume prior to the implant placement [3].The use of autologous block bone grafts prior to implant placement is another well-established surgical procedure for horizontal alveolar ridge augmentation.

The aim of this research is to present a novel surgical technique that was applied on 5 patients with atrophied alveolar bone to perform horizontal augmentation at the posterior maxilla with the use of partial alveolar split osteotomy and inter-positional block bone grafts.

\section{MATERIAL AND METHODS}

Systemically healthy five patients referred to the clinic for implant treatment. All of the patients have severely resorbed posterior maxillary bone and needed a pre-implant surgery to graft or augment the area prior to the implant placement. At first, the ridge-split technique was applied to the posterior maxilla. Then, a horizontal augmentation procedure was performed with a bone block from the ipsilateral mandibular ramus. Implants were placed at the fourth month of the surgery. Prosthetic rehabilitation was completed after the osseointegration of the implants.

Patient selection criteria for the proposed surgical technique:

1.Patients with no systemic health problems

2.Patients with no tobacco addiction

3.The regions with low bone quality and low bone density (optionally maxillary bone)

4.Minimum 2-3 mm of bucco-palatal bone width

5.Minimum $6 \mathrm{~mm}$ of vertical bone distance to maxillary sinus (to place short implants avoiding sinus floor elevation)

6. All kinds of gingival bio-type

7. Sufficient interocclusal distance with no vertical loss
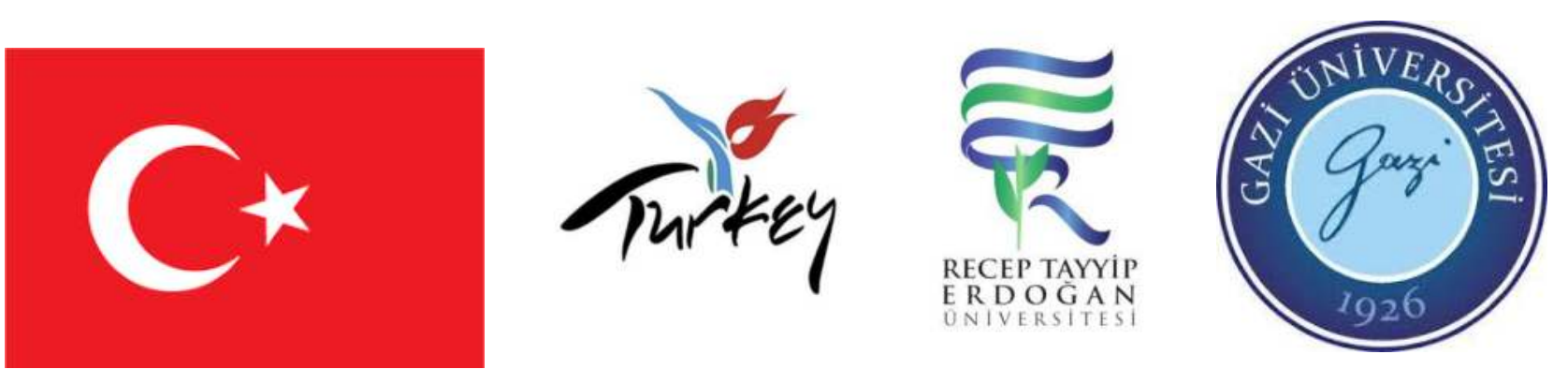

\section{RESULTS}

There were not any major complications at the donor or the recipient sites occurred during the post-operation period in any of the patients. The healing went uneventful. Radiological control was undertaken periodically after the surgeries. The stability of the implants was measured with the use of a hand wrench device before prosthetic loading and the torque values are considered indicative of the absence of implant mobility [4].

CBCT scans were taken from each patient during the follow-up control at the end of the first year. There were not significant bone loss around the implants. The success criteria for endosteal implants, defined by Buser et. al, was achieved in this one-year follow-up. Also, there were not any negative outcomes such as exudate, redness, swelling around the soft tissues, or gingival pockets greater than $4 \mathrm{~mm}$. All the implants supported mastication function.

The patients has been attending follow-up controls for over 24 months now. The regular controls are being held for the purpose evaluating the long-term prosthetic success of the operation.
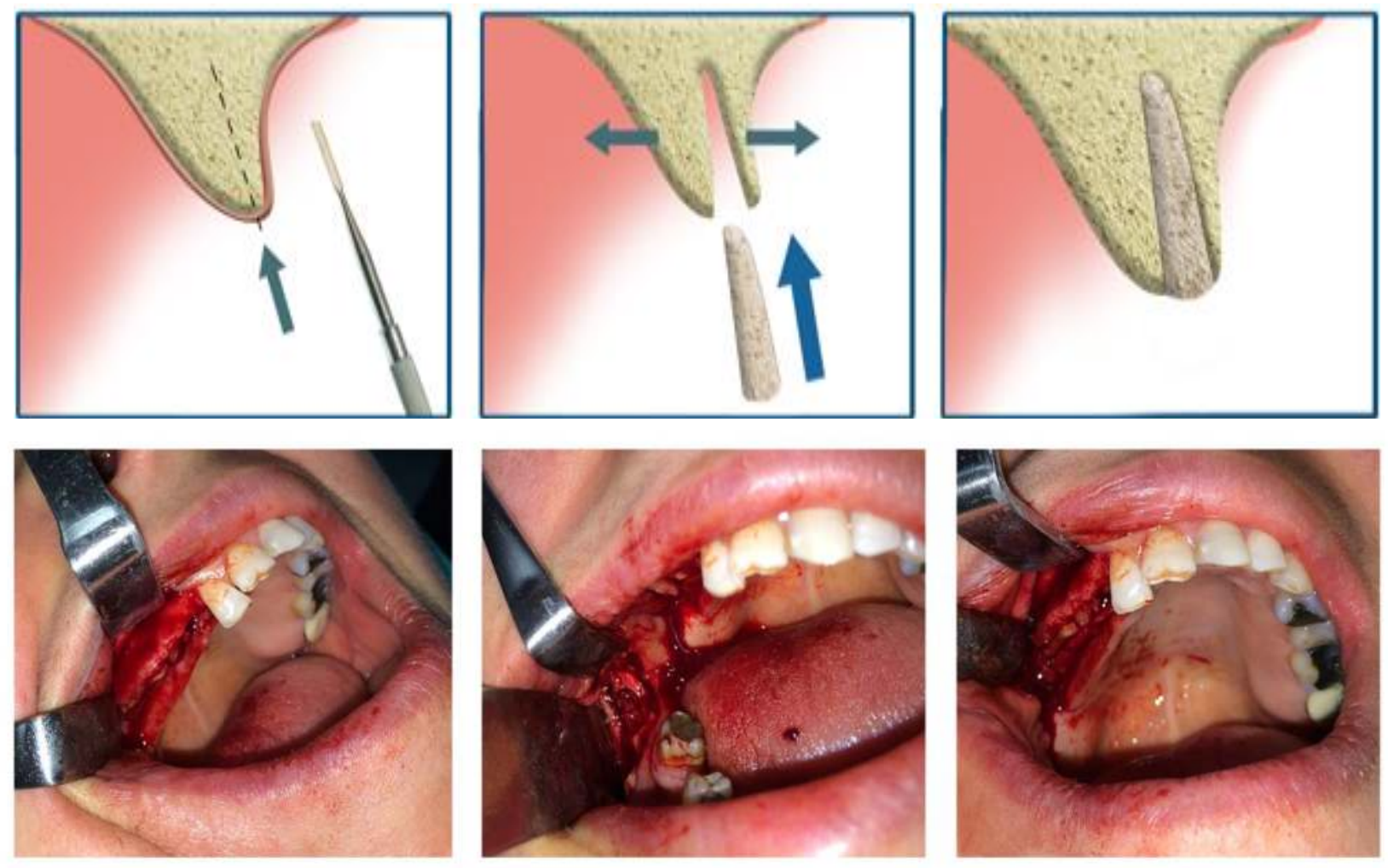

\section{CONCLUSION}

This novel technique provides a high-degree primary graft stability without the use of any fixation appliances. It enables the vascularization of the transplanted bone and increases the bone quality on the posterior maxilla, leading to a higher primary stability during the implant placement.

The presented case showed the benefits of a promising treatment option for horizontal ridge augmentation of the posterior maxilla prior to implant placement. Further studies and patient applications will provide more data from which to evaluate the benefits of the technique.

\section{REFERENCES}

1.Moraschini $V$ et. al Evaluation of survival and success rates of dental implants reported in longitudinal studies with a follow-up period of at least 10 years: a systematic review. Int J Oral Maxillofac Surg 2015;44:377-388.

2.Araújo MG et. al Dimensional ridge alterations following tooth extraction. An experimental study in the dog. J Clin Periodontol 2005;32:212-218.

3.Misch CE. Density of bone effect on treatment plans, surgical approach, healing, and progressive boen loading. Int J Oral Implantol 1990;6:23-31.

4.Etoz OA et. al New method to increase inter-alveolar height with preservation of crestal cortical bone for implant treatment. J Oral Implantol 2014;40:601-602. 